\title{
LINEAR INDEPENDENCE IN ABELIAN GROUPS
}

\author{
MARY-ELIZABETH HAMSTROM
}

Alexandroff and $\mathrm{Hopf}^{1}$ offer a proof of the following theorem. ${ }^{2}$ If $U$ is a sub-group of an Abelian group $J$ and $m$ is an integer such that $m=0$ or $m \geqq 2$, then $r_{m}(J) \geqq r_{m}(U)+r_{m}(J-U)$. The proof is incorrect and the following example shows that the theorem is, in fact, not true.

ExAmple 1. Let $J$ be the group of integers $\bmod 4$, and $U$ the subgroup generated by $2 ; r_{2}(J)=1, r_{2}(U)=1, r_{2}(J-U)=1$.

The proof referred to is correct if $m=0$, and the authors, in fact, prove that $r_{0}(J)=r_{0}(U)+r_{0}(J-U)$. In what follows we shall assume this, and that all groups considered are finitely generated and Abelian. ${ }^{3}$

Theorem 1. If (1) the group $V=\sum_{j=1}^{r} N_{j}$ is the direct sum of indecomposable cyclic sub-groups, $N_{j}$, (2) $m=p_{1}^{\alpha_{1}} \cdot p_{2}^{\alpha_{2}} \ldots \ldots p_{n}^{\alpha_{n}}$, where for each $i, p_{i}$ is a prime number, and (3) for each $i, q_{i}$ is the number of the $N_{j}$ whose orders are divisible by $p_{i}^{\alpha_{i}}$, then $r_{m}(V)=k$, where $k$ is the least of the $q_{i .}{ }^{4}$

Proof. We can assume, without loss of generality, that $q_{1} \leqq q_{2}$ $\leqq \cdots \leqq q_{n}$. The problem, then, is to show that $r_{m}(V)=q_{1}=k$. Clearly, $V$ is a direct sum $V=\sum_{1}^{k} V_{i}+\sum_{k+1}^{l} V_{i}$ where for each $i$, $V_{i}$ is cyclic and (1) if $1 \leqq i \leqq k, V_{i}$ has order divisible by $m$, (2) if $k+1 \leqq i \leqq l, V_{i}$ has order not divisible by $p_{1}^{\alpha_{1}}$. For each $i$, let $x_{i}$ be a generating element for $V_{i}$. The $x_{i}$ form a basis for $V$ and $k \leqq r_{m}(V)$.

Suppose $y_{1}, y_{2}, \cdots, y_{k+1}$ is a set of $k+1$ elements in $V$. For each $i$,

$$
y_{i}=\sum_{j=1}^{k} a_{i j} x_{j}+\sum_{j=k+1}^{l} a_{i j} x_{j}
$$

For each $i$, the order of $\sum_{j=k+1}^{l} a_{i j} x_{j}$ is not divisible by $p_{1}^{\alpha_{1}}$, so there exist constants $r_{1}, r_{2}, \cdots, r_{k+1}$, no one of which is divisible by $p_{1}^{\alpha_{1}}$,

Received by the editors May 9, 1949 and, in revised form, May 8, 1950.

${ }^{1}$ P. Alexandroff and H. Hopf, Topologie, Berlin, 1935, p. 572.

2 The elements $x_{1}, x_{2}, \cdots, x_{n}$ of an Abelian group $J$ are said to be linearly independent $\bmod m$ if $\sum_{i=1}^{m} a_{i} x_{i}=0$, where the $a_{i}$ are integers, implies that $a_{i} \equiv 0 \bmod m$ for each $i$. The rank mod $m$ of $J, r_{m}(J)$, is the largest integer $n$ such that there exists a set of $n$ elements in $J$ which are linearly independent $\bmod m ; r_{0}(J)$ denotes ordinary rank.

${ }^{3}$ We shall assume, further, that $r_{m}(J)$ is finite. Theorems 2 and 3 of this paper are true without the condition that $J$ be finitely generated. This follows without too much difficulty from the proofs of these theorems.

${ }^{4} \mathrm{We}$ assign order 0 to infinite cyclic groups. 
such that for each $i, r_{i} \sum_{j=k+1}^{l} a_{i j} x_{j}=0$. Clearly, for each $i$,

$$
r_{i} y_{i}=r_{i} \sum_{j=1}^{k} a_{i j} x_{j} \neq 0 \text {. }
$$

Since we have $k+1$ equations in $k$ indeterminates, there exist constants $t_{1}, t_{2}, t_{3}, \cdots, t_{k+1}$, relatively prime, and such that for each $j, \sum_{i=1}^{k} t_{i} a_{i j}=0$. Therefore,

$$
\sum_{1}^{k+1} t_{i} r_{i} y_{i}=0 .
$$

At least one of the $t_{i}$ is not divisible by $p_{1}$. Therefore, at least one of the $t_{i} r_{i}$ is not divisible by $p_{1}^{\alpha_{1}}$, and is, therefore, not divisible by $m$. It follows that the $y_{i}$ are linearly dependent mod $m$. Therefore, $r_{m}(V)=k$.

The following are direct consequences of the above proof.

COROLLARY 1. If $r_{m}(J)=k$ there exists a set of $k$ linearly independent elements mod $m$ each element of which has order $m$ or 0 .

CoRollary 2. The rank of $J, r_{0}(J)$, is the number of the $V_{i}$ whose order is 0 , and if $R_{m}(J)$ denotes the number of the $V_{i}$ whose order is divisible by $m$, but is not 0 , then $r_{m}(J)=r_{0}(J)+R_{m}(J)$.

Theorem 2. If $J$ is a finitely generated Abelian group and $U$ is a sub-group with division ${ }^{5}$ of $J$, then $r_{m}(J)=r_{m}(U)+r_{m}(J-U)$.

Proof. By Corollary 2 above, $r_{m}(U)=r_{0}(U)+R_{m}(U)$. Since $U$ is a sub-group with division, each element of $(J-U)$ has order 0 , and $r_{m}(J-U)=r_{0}(J-U)$. Clearly, $R_{m}(U)=R_{m}(J)$. Therefore, since $r_{m}(U)$ $+r_{m}(J-U)=r_{0}(U)+R_{m}(U)+r_{m}(J-U), r_{m}(U)+r_{m}(J-U)=r_{0}(U)$ $+r_{0}(J-U)+R_{m}(J)=r_{0}(J)+R_{m}(J)=r_{m}(J)$.

The same authors ${ }^{6}$ attempt to prove that if $p$ is a prime number and $U$ is a sub-group of the group $J$, then $r_{p}(J) \leqq r_{p}(U)+r_{p}(J-U)$. The proof is incorrect. I offer in its place a valid proof.

TheOREM 3. If $p$ is a prime and $U$ is a sub-group of the group $J$, then $r_{p}(U)+r_{p}(J-U) \geqq r_{p}(J)$.

Proof. There is a set of $r_{p}(U)$ elements of $U, x_{1}, x_{2}, \cdots, x_{r_{p}(U)}$ linearly independent $\bmod p . R_{p}(U)$ of these form a basis for the subgroup of $U$ consisting of all elements in $U$ of order $p$. There is a set

${ }^{5}$ The sub-group $U$ of $J$ is said to be a sub-group with division of $J$ provided $p x \in U, p \neq 0$, implies that $x \in U$.

- Alexandroff and Hopf, loc. cit., p. 573 
$y_{1}, y_{2}, \cdots, y_{k}$ of elements of $J$ such that (1) for each $i, y_{i}$ is of order $p$, (2) $k=R_{p}(J)-R_{p}(U)$, and (3) $x_{1}, x_{2}, \cdots, x_{r_{p}(U)}, y_{1}, y_{2}, \cdots, y_{k}$ is a basis for the sub-group of $J$ consisting of all elements of order p. Clearly, $U+y_{1}, U+y_{2}, \cdots, U+y_{k}$ are independent $\bmod p$ in $J-U$, and $R_{p}(J-U) \geqq k$. Now,

$$
\begin{aligned}
r_{p}(U)+r_{p}(J-U) & =r_{0}(U)+R_{p}(U)+r_{0}(J-U)+r_{p}(J-U) \\
& \geqq r_{0}(J)+R_{p}(U)+k \\
& =r_{0}(J)+R_{p}(J)=r_{p}(J) .
\end{aligned}
$$

Example 1 shows that the inequality can hold. The following example shows that Theorem 3 is not true for composite numbers.

EXAMPLE 2. Let $J$ be the group of integers $\bmod 12$, and $U$ the sub-group generated by 2 . Then, $r_{4}(J)=1, r_{4}(U)=0, r_{4}(J-U)=0$.

It can be proved by methods quite similar to those in this paper that the equality in Theorem 3 holds if and only if $p U$ equals the common part of $U$ and $p J$, but this lies outside the purpose of this paper.

UNIVERSITY OF TEXAS 